\section{ACTUALIDAD \\ DE LA FENOMENOLOGÍA: \\ LA FENOMENOLOGÍA DE LA VIDA DE RENAUD BARBARAS}

\author{
Mariana Larisson \\ Universidad de Buenos Aires
}

\begin{abstract}
In recent years we have seen a resurgence of a number of concepts connected to the notion of life, as well as to the several problematic that have their origin in this notion. Such problematic have come to be fashionable in fields such as science, politics, culture or in recent developments in economics and technology. Against this historical background, philosophical reflection on the phenomenon of life has not been, however, undertaken. In this context, the appearance of a philosophical attempt that presents itself as a phenomenology of life is of immediate interest and relevance. Such is the work of Renaud Bárbaras entitled Introduction à une phénoménologie de la vie (2008). The following analysis focuses on this text and attempts to bring to light its great problematic and possible implications.
\end{abstract}

KEY WORDS: Phenomenology, Life, Intentionality, Movement, Experience, Desire.

En el comienzo del nuevo milenio, un tema crucial acecha en el horizonte de múltiples preocupaciones, tema que sin embargo podríamos referir a un solo nombre: la vida. Múltiples preocupaciones que van desde el reclamo por nuevas definiciones de la vida como consecuencia del desarrollo vertiginoso de la biología durante el siglo pasado y del desarrollo de nuevas tecnologías para suplantar las condiciones de vida existentes hasta hoy, pasando por los resultados efectivos de un avance del capital económico que no reconoce la vida como límite para su propia y anónima autoconservación. Problemas tales como los nuevos escenarios de la política internacional, que comienzan a proclamar una nueva distribución del territorio mundial en nombre de valores como la conservación del medio - del mismo modo que, en otras ocasiones, en nombre de otros igualmente indiscutibles valores como la humanidad, la civilización o la libertad-, y que corren astutamente en paralelo a un urgente y auténtico reclamo por una modificación global de las condiciones de explotación de los recursos naturales -de

\section{THE PRESENT OF THE PHENOMENOLOGY: THE PHENOMENOLOGY IN THE LIFE OF RENAUD BARBARAS}

RESUMEN: En los últimos años hemos asistido al surgimiento de toda una serie de conceptos ligados a la noción de vida y a las problemáticas que de ella se derivan. Las mismas que, hoy en dia, se encuentran en boga en ámbitos tan diversos como la ciencia, la política, la cultura o los nuevos desarrollos económicos y tecnológicos. Sin embargo, y con este contexto histórico de fondo, la reflexión filosófica sobre el fenómeno de la vida resta aún por hacerse. En este sentido, la aparición de una filosofía que se presenta a sí misma como una fenomenología de la vida cobra una actualidad e interés inusitados. Tal es el caso de la reciente obra de Renaud Barbaras Introduction à une phénoménologie de la vie (2008), de la que se ocupa el presente escrito, en el que intentaremos poner de relieve la problemática que el texto revela y sus posibles derivaciones.

PALABRAS CLAVE: Fenomenología, vida, intencionalidad, movimiento, experiencia, deseo.

los que África representa hoy uno de los más terribles testimonios-. No cabe duda: la interrogación sobre la vida se impone, de manera ineludible, a la reflexión y a la discusión filosófica.

Más aún, podría pensarse que el espacio que antes fuera ocupado por otros grandes conceptos - grandes en el sentido que albergaban un campo hermenéutico vastísimo de problemas-, fuera tomado hoy, de manera cada vez más insistente, por el fenómeno de la vida. $Y$, como sucede precisamente con los grandes y polémicos conceptos, nadie está muy seguro de a qué refiere este nombre.

Es así, sumergidos en este contexto histórico, como la aparición de un pensamiento que toma la forma de una fenomenología de la vida cobra una actualidad inusitada. La reciente obra de Renaud Barbaras, Introduction à une phénoménologie de la vie', nos ofrece justamente la ocasión de pensar con seriedad sobre el sentido de ser de aquello que llamamos vida, y sobre el modo en que nos 
interpela desde el punto de vista del pensamiento y de la acción en tanto que filósofos.

\section{INTRODUCCIÓN A UN PENSAMIENTO DE LA VIDA}

La obra del filósofo francés Renaud Barbaras comenzó con un fuerte trabajo crítico en torno a la obra de MerleauPonty, de quien es uno de los grandes comentadores y a quien debemos, en una medida tal vez inconmensurable, la actualización, difusión y seriedad de los estudios merleaupontianos. Interesado en toda la historia del movimiento fenomenológico, Barbaras es, sin embargo y ante todo, un filósofo. En Introduction à une phénoménologie de la vie, obra de reciente aparición, el fenomenólogo francés realiza un trabajo de interpretación absolutamente original del principio fundador de la fenomenología, el $a$ priori universal de la correlación intencional, que reconfigura completamente el sentido y la función de la empresa fenomenológica. A partir de un cuidadoso trabajo de despliegue de los conceptos (en el que retoma y dialoga con la tradición filosófica de la que forma parte y a la que, al mismo tiempo, contribuye a construir), Barbaras reconduce el problema de la correlación al de la vida y al del deseo, dando lugar de este modo a lo que podríamos llamar una "metafísica del deseo", que permite realmente volver a pensar las categorías tradicionales de la ontología moderna y contemporánea.

Dada la magnitud, complejidad y novedad de Introduction à une phénoménologie de la vie, nuestra intención en este ensayo se limita a una presentación del pensamiento barbarasiano, seguida de algunas conclusiones que, tal sería nuestro objetivo, puedan abrir el debate y ser tomadas como índices de una tarea a realizar.

\section{INTRODUCCIÓN A UNA FENOMENOLOGÍA DE LA VIDA}

El punto de partida de esta obra es, como lo hemos anticipado, la pregunta que preside el movimiento fenomenológico como tal, acerca de un a priori básico: el de la correlación entre el ente trascendente y sus modos subjetivos de donación. El punto neurálgico sobre el que se centrarán y confrontarán, en el seno de la fenomenolo- gía, su fundador y sus seguidores, es el estatuto del sujeto de esta correlación. Según Barbaras, este sujeto abre una nueva dimensión, un nuevo sentido de ser, sobre el que la fenomenología impone desde su fundación el deber de interrogar y que, sin embargo y como esta obra se encargará cuidadosamente de mostrarlo, no ha dejado de hacer a partir de las herramientas equivocadas. En otros términos, el problema del sujeto de la correlación ha sido abordado a partir de categorías propias de una tradición metafísica dualista, a la que la fenomenología no adeuda ningún compromiso esencial, por lo que es necesario dejarlas de lado y comenzar -iuna vez más!- desde el principio.

Sin embargo, sugiere nuestro autor, si bien el compromiso de la fenomenología con la tradición dualista no es esencial, tampoco es sin embargo azaroso: la ontología de la época moderna se encuentra orientada por una represión fundamental de la vida, cuyos resultados ella misma, como corresponde a una represión à la lettre, desconoce y sabe al mismo tiempo. Se desconoce como lo que es: una ontología de la muerte; se sabe en lo que niega: el retorno permanente del vocabulario de la vida que nunca es, curiosamente, tomado al pie de la letra.

Con la precisión de una intervención analítica, Barbaras tomará en cambio el camino indicado por este retorno. En otras palabras, si, de una forma u otra, todos los fenomenólogos se han confrontado en algún momento al problema de la vida pero sin interrogarla por sí misma, habrá pues que hacer lo inverso y preguntarse por lo no dicho en estos acercamientos.

De esta forma surge una hipótesis que presidirá la obra: ¿y si acaso el sentido de ser del sujeto de la correlación, sobre el que nadie puede responder si no es desde categorías dualistas, fuera el de la vida misma? ¿No es acaso el sujeto, de manera esencial, aquel que dirige el aparecer del mundo al que al mismo tiempo pertenece? Pues bien, el término "vivir"2 bien parece responder a esta doble exigencia: vivir en el sentido de "estar en vida", y vivir en el sentido de "hacer la experiencia de algo", y por tanto relacionarse esencialmente a otra cosa que sí mismo. En otros términos, ser un viviente y tener conciencia. En este sentido, continúa Barbaras, el vivir es el modo en que se articulan la univocidad y la equivocidad del sujeto de la correlación: equivocidad en tanto su sentido de ser lo 
distingue de los otros entes no vivientes, univocidad en tanto comparte este sentido.

Todo el problema -y "todo" tiene aquí la extensión de la obra entera-, es determinar cómo es posible la equivocidad y la univocidad del vivir considerado como el sentido de ser del sujeto.

En cualquier caso, la ecuación ya está hecha: si la fenomenología se ocupa del ser del sujeto, la fenomenología es siempre fenomenología de la vida. Si todos los fenomenólogos de la tradición han abordados la vida pero la han dejado escapar como problema, es que en todos ellos se constata la adhesión irrefleja a lo que Barbaras llama, siguiendo a H. Jonas, "ontología de la muerte". En efecto, y como parte de la tesis acerca de una represión fundamental de la vida, se encuentra la idea según la cual la noción de vida que atraviesa la época moderna se encuentra subordinada a una ontología de la muerte. A partir de ésta, en lugar de pensarse la vida a partir de ella misma, se la piensa a partir de la muerte, que pasa a ser el fenómeno natural a partir del cual deberá ser tomado el sentido de ser de lo que es, y la vida será un problema que es necesario explicar.

La consecuencia de una posición tal es la consideración de la vida como supervivencia y conservación de sí, dado que lo contrario es la desaparición de sí en la tumba terrestre. Para salir de esta orientación, y porque estamos en el camino de una fenomenología de la vida, Barbaras propone una epojé de la muerte. A través de ella, la pregunta por el sentido de la vida se separa de la pregunta por sus condiciones de existencia: una fenomenología de la vida debe interrogarse por el sentido de ser de la vida en tanto es el aspecto común de la correlación, y en tanto es el lugar en el seno del cual algo puede diferir como sujeto de esta misma correlación.

De este modo, la primera parte de la obra se inicia con la pregunta por el vivir, en tanto este fenómeno engloba una equivocidad esencial: ¿qué es la vida en tanto ella puede dar cuenta del ser viviente y del experimentar?

Pues bien, en la posición misma de la pregunta aparece un primer criterio para su respuesta: ésta debe buscarse en la experiencia misma del vivir, pues el conocimiento de la vida impone el reconocimiento de la vida. Este re- conocimiento impone además que sólo un viviente pueda reconocer a otro viviente, y que, por tanto, la vida implique la exteriorización de manera esencial.

A partir de estos criterios, la búsqueda del sentido de ser de la vida será la ocasión de un recorrido crítico por las obras de Henry, de Heidegger, Merleau-Ponty y Patocka. Si Henry tiene razón al identificar conciencia y vida, se equivoca al pensar la vida como pura inmanencia. Si a su vez Heidegger tiene razón en señalar la vida como el lugar de la articulación entre existencia y subsistencia, tal vez se equivoca en utilizar estos mismos conceptos.

En efecto, Barbaras advierte que, al abrir Heidegger la interrogación sobre el Dasein como el modo de ser de un ente particular que, siendo ente difiere al mismo tiempo del ente, limita su interrogación al continuar con la problemática distinción entre existencia y presencia subsistente, en la que quedará atrapada su propia concepción de la vida.

Si la existencia es propia del Dasein, no puede atribuirsela al viviente como tal, que excede el ámbito de Dasein. Ella debe ser sólo el punto de partida para un acercamiento privativo a la vida, una zoología privativa en la que la vida sería el Dasein menos algo. Pero, señala con pertienencia Barbaras, si la existencia sólo se dice del Dasein, ella tiene que dar acceso a otro tipo de ser, $y$, en este sentido, debe producirse una alteración en el pasaje del Dasein a la vida, y no una mera sustracción privativa, lo que pone en jaque el proyecto mismo de una zoología privativa. En definitiva, partiendo del Dasein, Heidegger no alcanza finalmente una interrogación sobre la vida como tal. Lo que nos deja una lección que Barbaras explicita: la cuestión no debe plantearse a partir del Dasein, para llegar a la vida, sino preguntarse cómo pensar la vida en tanto ella da lugar a algo así como el hombre, o Dasein.

En efecto, lo que la distinción heideggeriana entre existencia y presencia subsitente no permite entender es cómo se articulan, en el mundo, la existencia y la subsistencia. Pues bien, señala Barbaras, para comenzar, hay que pensar la dimensión de intramundaneidad del Dasein, es decir, el cuerpo.

Lo que nos lleva a un sintético pero brillante pasaje por la obra de Merleau-Ponty, fenomenólogo del cuerpo por excelencia. Desde la perspectiva de Barbaras, Merleau- 
Ponty abre, hacia el final de su vida, el camino para pensar con claridad el problema del sujeto de la correlación como simultaneidad del estar en vida y del experimentar. Según esta lectura, la noción de chair designaría la unidad misma del sentir y de ser del mundo, cuyo sujeto no es el cuerpo (que sólo es un derivado) sino el mundo mismo. El problema es que esta noción de chair es lo suficientemente ambigua como para dar lugar tanto a un monismo ontológico como a una nueva dualidad en el seno del sentir.

Si Merleau-Ponty tiene razón en buscar un sentido de ser del sujeto que incluya la sensibilidad, se equivoca en buscarlo en la carne y no en la vida. Es por la vida por lo que hay cuerpo, y la vida es existencia encarnada. Patocka se revela así la guía de un pensamiento de la existencia que se colocaría más allá de Husserl, Heidegger y Merleau-Ponty.

Según Barbaras, Patocka ha visto con mucha claridad que la subjetividad es tan sólo una función que se define por ser aquello a quien aparece lo que aparece y bajo tres condiciones: ser a partir de la correlación misma, ser co-condicionante (es decir, estar en relación directa con el otro polo de la relación, el mundo como totalidad) y ser a su vez englobado, parte de la totalidad con la que se relaciona. De este modo, la alternativa de Barbaras es clara: si Husserl identifica el campo fenomenal con un ente particular -la conciencia-; si Heidegger piensa el Dasein como posibilidad pero sin un cuerpo para realizarla; y si Merleau-Ponty piensa el cuerpo propio pero limitado a su "propiedad", es entonces en la definición formal del sujeto de la correlación dada por Patocka donde hay que buscar una nueva vía de investigación.

Así, al definir la corporalidad como conjunto de posibilidades no elegidas de las cuales la esencial es la capacidad de moverse, Barbaras muestra cómo el filósofo checo coloca el problema en una nueva dirección: el movimiento permite pensar la unidad y la equivocidad del sujeto. Por ser el lugar de despliegue del mundo, es del mundo; pero por ser la negación de toda presencia subsistente, es distinto de los otros entes. A partir de una relectura del análisis aristotélico del movimiento, cuyo modo esencial sería el movimiento ontogenético, Patocka emprende un análisis del movimiento de la existencia en tanto efectividad de la vida en sus diversos momentos.
Sin embargo, Barbaras intenta mostrar en este punto que, a pesar del enorme paso dado en el sentido de la investigación, Patocka olvida en el camino la centralidad del cuerpo y la equivalencia de la vida y la existencia: el movimiento descrito por éste, que correspondería a la existencia humana, no necesariamente corresponde a la vida, y el cuerpo es tomado como una condición de efectuación pero no como un elemento esencial de la existencia.

Reformulando las cosas una vez más, la pregunta se vuelve: ¿cómo pensar la vida para que ésta pueda dar cuenta de la existencia en todos sus niveles? Esta pregunta orientará, pues, la segunda parte de la obra, ocasión de reflexionar con y a partir de figuras tan disímiles como Bergson, Ruyer, Jonas y Rilke. Es decir, un metafísico, un filósofo de la ciencia, un pensador de la naturaleza y la técnica, y un poeta; no hace falta ya repetir que la vida ama ocultarse.

Comenzar la indagación a través de Bergson parece en principio razonable, en la medida en que es el suyo un pensamiento de la vida a partir de la duración -y por tanto asimilable a la conciencia- como unidad dentro de la cual los mortales, como puntos de pasaje, permiten la continuidad de una vida creadora. Sin embargo, señala Barbaras, el problema de este pensamiento es la división metafísica fundamental entre materia y memoria que dará lugar a una división en el seno mismo de la vida. Tal como lo muestra Barbaras, la vida, extranjera a la materia en tanto principio positivo, sólo puede degradarse en supervivencia en su contacto con la materia. Desde este punto de vista, Bergson se equivoca al pensar la vida como un principio positivo, pero tiene razón al pensar al hombre como una privación en el seno de la vida. Lo que nos obliga a recordar una vez más que la vida no puede excluir la exterioridad a sí sino que, por el contrario, tiene que ser ella misma exterioridad ${ }^{3}$.

Barbaras encuentra en Ruyer un acercamiento completamente original de la vida y de la extensión. En efecto, Ruyer identifica por primera vez la vida con la conciencia y la extensión. Según Ruyer, el tejido viviente es una realidad unitaria y conciente, que se delimita a sí misma como una unidad por la conciencia misma que la sobrevuela. Este tipo de realidad es denominada por Ruyer "dominio de sobrevuelo". En este sentido, lo que habitualmente entendemos por conciencia sería un tipo de conciencia secundaria, propia al cerebro en tanto tejido viviente. La 
conciencia primaria, en sentido estricto, se identifica con el tejido viviente sin más. El problema, señala Barbaras, es, por un lado, que esta filosofía desemboca en un metafísica de la mónada: lo que se gana en extensión se pierde en relación, pues la "unidad dominante" que piensa Ruyer sólo se relaciona con lo que subrevuela, es decir, consigo misma. Por otro lado, la identificación entre conciencia y extensión produce una reducción de la extensión a la conciencia, en la medida en que el espacio se vuelve superficie y pierde la dimensión de profundidad que lo define, y que escapa esencialmente a la conciencia. De hecho, como intenta mostrar Barbaras desde el comienzo de su investigación, el problema es pensar la conciencia en tanto se relaciona con algo que la supera y que la excede, estableciendo así una relación esencial a la alteridad.

En este sentido, Jonas muestra, aunque al precio de muchos problemas, un aspecto fundamental del viviente: que su relación con la exterioridad presupone una incompletud esencial, puesto que la relación a lo otro de sí supone una falta de sí. En el caso de Jonas, el problema que señala Barbaras es que esta incompletud se describe en los términos inadecuados. Según el pensador alemán, lo que define al viviente es el metabolismo considerado como producción de una forma unitaria desplazada respecto de su materia diversa. Es la necesidad de la materia la que abre al viviente, según Jonas, la trascendencia del mundo.

Pues bien, Barbaras va a mostrar precisamente lo contrario: la necesidad no abre sino que supone la trascendencia del mundo. La necesidad apunta a un objeto determinado, susceptible de satisfacerla. Ahora bien, antes del acceso a lo determinado, hay que suponer un acceso, un movimiento hacia lo indeterminado, dentro del cual un objeto puede ser definido.

De esta forma, la operación barbarasiana hasta el momento podría resumirse de la siguiente manera: dar cuenta de la vida es encontrar la unidad primordial del vivir y del experimentar, y es también dar cuenta de la dimensión en la cual estos términos pueden divergir. Decir en qué difiere el experimentar del simple estar en vida, y en qué difiere el animal del hombre y de la planta, no es decir en qué es diferente, sino cómo es posible que difiera. Pero al decir cómo es posible, la diferencia toma una forma determinada. El razonamiento es entonces el siguiente: si la vida es más amplia que sus diferencias y debe contenerlas, pero de un modo que no sean diferencias de especie ni una continuidad gradual, esta diferencia sólo puede ser la del límite. La forma determinada de la diferencia se presenta así como límite, límite que es interpretado como privación o, incluso, como represión.

La elucidación de esta dimensión será iluminada por la 0ctava elegía de Duino, de Rilke. Barbaras seguirá el poema, de la mano de Munier, en el que se describe la oposición del hombre y el animal a partir de la oposición entre lo Abierto y el mundo.

Siguiendo la poética de Rilke, Barbaras describe lo Abierto como el ámbito en el que residen todas las cosas -antes de su determinación como estas cosas-, mientras que la conciencia y el mundo (que constituyen la humanidad) nacen de un retroceso frente a esta completa apertura. En este sentido, si lo Abierto es un puro llamado, la conciencia y el mundo nacen de un movimiento de oposición a este llamado, a partir del cual lo Abierto aparece, oculto bajo la forma del mundo. La forma del mundo revela así una mirada, la del hombre, que se distingue de la mera visión del animal. Un tal movimiento de oposición no es otro que la oposición al impulso originario hacia lo Abierto, oposición de la que nacen la conciencia y el mundo, y en el que Barbaras encuentra un movimiento de represión originaria. En este sentido, la humanidad es represión de la vida.

Pues bien, a través de Rilke, Barbaras encuentra esta dimensión negativa dentro de la cual la transitividad de la vida será posible. Esta represión, lo es de lo Abierto en el doble sentido del genitivo: lo Abierto es lo que falta y él que falta. Lo Abierto es falta. Así, lo más originario aquí es la negación originaria de la que proceden todos los términos en juego: lo que la humanidad niega es una plenitud que nunca tuvo lugar. La transitividad fundamental de la vida significa así una relación a lo que ya siempre ha faltado $y$, de este modo, ella puede hacerse vida fenomenalizante, es decir, vida conciente.

La existencia humana, caracterizada por la conciencia, procede de una limitación en el seno de la vida que Barbaras caracterizó como represión. La tercer y última parte de la obra tiene como fin determinar el sentido de esta limitación, el sentido de la vida en tanto movimiento de auto-limitación y del Ser que puede corresponderle.

ARBOR CLXXXV 736 marzo-abril [2009] 303-311 ISSN: 0210-1963

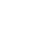


¿Qué ha quedado del sujeto, desde el punto de vista conceptual, una vez reinterpretado su lugar a partir de una vida definida como movimiento del vivir?

Pues bien, ha quedado un sujeto de la experiencia, entendida no como experiencia de algo, sino como la pura experiencia que somos, en el sentido dado por Patocka a esta expresión.

Desde esta perspectiva, la experiencia no es pensada como recepción de "algo", sino como posibilidad de toda recepción. La experiencia se revela así un movimiento negativo que impide que cualquier dato la colme, pues en la posición de algo, ella se transborda inmediatamente a lo que lo excede.

En términos que atravesarán la última parte de la obra, lo que aqui se pone en juego es una dialéctica de lo finito y lo infinito: lo dado, un objeto, se presenta en su finitud ante un sujeto como lo otro de su propia infinitud vacía de todo contenido. En este sentido, el movimiento de la vida es el lugar donde una dialéctica de lo finito y de lo infinito puede darse pero que, precisamente por esto, él supera.

Lo importante en el marco del despliegue barbarasiano del problema es que el sujeto, como sujeto de la experiencia o movimiento en el seno del vivir, ha perdido toda posibilidad de reificación y ha quedado reducido a una negatividad, a un no-ser.

La actividad filosófica, en este sentido, se revela también como esencialmente vital, pues ella no es otra cosa que el movimiento infinito de búsqueda de un ser que nunca podrá satisfacerla y que no se detiene jamás. En este sentido, ella revela la esencia del vivir: no por lo que dice, no por su contenido, sino por su actividad misma. Ella actúa la vida, y, sólo en este sentido, lejano a su propia conciencia, la "representa".

De este modo llegamos al corazón mismo de la filosofía barbarasiana: si el movimiento del vivir es esta dimensión en la que se constituyen mutuamente finito e infinito, la esencia del vivir adquiere el sentido del deseo. Relacionándose a la trascendencia en el modo de la insatisfacción delante de lo finito, el deseo relaciona el mundo con lo Abierto.
Una fenomenología de la vida parece fundarse asi en una meta-física del dese $0^{4}$. Si el deseo es la esencia del vivir, y el vivir es la dimensión común de la correlación, el deseo -como el vivir- es lo que define al sujeto de la correlación como diferencia tanto como al objeto. Es aquello que los hace posibles. En este sentido, el deseo es la esencia del sujeto de la correlación en tanto es la modalidad efectiva de la intencionalidad. 0 , dicho de otro modo, la intencionalidad es un modo del deseo.

En efecto, lo que intentará mostrar Barbaras es que el sujeto, antes de ser el sujeto de la intencionalidad, es sujeto del deseo, y es por esto por lo que puede haber algo como lo intencional (tanto desde el punto de vista del sujeto como del objeto). El deseo es búsqueda de si (del deseo) en lo otro. En lo otro, el deseo se vuelve lo que es. Pero su modo de ser es la ausencia: lo que revela lo otro es la falta de sí, el hecho de que él no es, y, en este sentido, revelación de una decepción. El deseo se revela así como una actividad sobre lo otro que, al mismo tiempo, es el resultado. Al advenir como tal en la actividad, adquiere la forma de un padecer, de un experimentar. De este modo, el deseo puede ser definido como falta del sujeto, una vez más en el doble sentido del genitivo: la falta experimentada por este sujeto es falta de este sujeto mismo. Lo que Barbaras pone en discusión aquí es la noción misma de experiencia: el sujeto es deseo pues desear es experimentar, como falta activa, que es al mismo tiempo pasiva, pues es recepción pura -recepción de todo.

Ya lo sabemos, la correlación, como tal, es la relatividad mutua del ente y de sus modos subjetivos de donación. Este es el campo de la fenomenalidad. Para dar cuenta del mismo a partir de la vida y del deseo que la define, Barbaras debe pensar el deseo desde el punto de vista del campo fenomenal. Pero el fenomenólogo francés ve bien el riesgo que se presenta con esta tarea: pensar el campo fenomenal, que es el lugar mismo de la correlación, no debe implicar que la correlación como tal sea considerada como una realidad ontológicamente primera, y que la diferencia entre los polos co-relacionados sea anulada.

Desde nuestro punto de vista, éste es el mayor desafío metafísico de la filosofía barbarasiana: de Hegel en adelante, el problema no es comprender una unidad que se auto-ponga en sus momentos. El problema es pensar esta unidad de un modo que no recaiga en un Absoluto idéntico a sí mismo y ontológicamente primero respecto de sus momentos. El 
problema no es pensar un movimiento que proceda por negaciones para realizar su propio despliegue: el problema es un movimiento que, por definición, excluya toda la positividad definida como identidad, como coincidencia de sí a sí. En otras palabras, lo que Merleau-Ponty había Ilamado una dialéctica sin síntesis o hiperdialéctica. Sin embargo, el problema de Merleau-Ponty fue haber tomado la dimensión unitaria de la correlación y haberla ontologizado, proponiendo a través de la noción de chair un monismo metafísico en el que la diferencia pierde todo sentido.

Por el contrario, contra un monismo metafísico, Barbaras propone pensar el campo fenomenal a partir de un monismo metodológico que tome como irreductible la correlación.

Pues bien, para que dos realidades puedan ser relacionadas, es preciso que haya un ámbito en el que la relación sea posible. La condición o fondo en el cual dos cosas pueden ser puestas en relación, no puede ella misma ser una realidad del mismo tenor: la dimensión originaria sólo puede existir bajo el modo de "fondo", "suelo" o Mundo. Pues bien, si el Mundo es la Totalidad, la Proximidad originaria nombra la dimensión de investimiento primario, en relación al cual lo cercano o lejano cobran sentido.

La correlación es el vivir, es la vida quien une sujeto y mundo, y el vivir es deseo. Pues bien, el deseo es la instauración de una dimensión de intimidad que se revela originaria. En este sentido, el deseo no une dos realidades, sino que es la dimensión en la que una relación puede ser posible. Pero en tanto que deseo, la Proximidad es Distancia originaria, pues aquello que puede ser relacionado originariamente es una distancia irreductible.

La realidad trascendente es fenoménica, y en este sentido relativa al sujeto; por otro lado, la fenomenalidad implica la distinción entre el aparecer y lo que aparece. Esta diferencia en el seno de la fenomenalidad entre el aparecer y lo que aparece no es una diferencia ontológica: es, en términos barbarasianos, una reserva de manifestación, de profundidad. Así, esta diferencia es propia al movimiento del aparecer como tal, es un exceso sobre sí por el que el aparecer no se agota en lo que es.

Por esta razón, el deseo es el principio de la trascendencia, pues sólo hay exceso irreductible de lo dado en y para el deseo. Lo que significa que hay un modo de donación originaria en el deseo que no puede ser subsumido en un objeto de conocimiento, y sobre la cual éste es posible, y que es precisamente el objeto del deseo.

En este sentido, afirma Barbaras, sólo la eidética husserliana de la percepción da las bases para pensar este oscuro objeto del deseo, que está lejos del objeto en sentido tradicional. El error de la fenomenología fue identificar la estructura del aparecer a la de la objetividad, y perder así para siempre la posibilidad de comprenderla. Lo deseado, por esencia, no es objetivable y lo percibido, en el sentido de la institución originaria de lo dado, no es un acto de conocimiento.

La profundidad de la aparición es el mundo que se esconde y permite la aparición finita. La aparición finita, es el límite del mundo. Lo deseado no es una ausencia susceptible de volverse presente, sino la ausencia en el corazón de la presencia. Es porque hacemos la experiencia de una falta en el corazón del ser por lo que el deseo es al mismo tiempo exceso inagotable. Exceso del que surgirán también tiempo y espacio, exceso que es al mismo tiempo Inacabamiento originario del Ser. Asi formula finalmente Barbaras su principio metafísico de base: hay Inacabamiento, y el Deseo es uno de sus modos, el que permite el acceso originario al Ser como Inacabamiento.

En verdad, el deseo muestra que no existe alternativa entre dualidad o unidad. El sujeto y el mundo son el exceso del deseo, exceso sobre sus realizaciones y exceso de las realizaciones sobre sus apariciones. El exceso sólo se produce en la realización efectiva del deseo, y por tanto sujeto y mundo como diferencias se producen en la unidad -una unidad que es movimiento realizante y nunca completamente realizado-. Si el deseo es la esencia de la vida, su modo de existencia es el movimiento. Por tanto, el sujeto del deseo existe como movimiento, pues el movimiento no es una realidad, sino una existencia en el modo de la diferencia.

\section{Conclusiones}

Obra de una enorme complejidad, de la que esperamos haber dado una idea justa, Introduction à une phénomé- 
nologie de la vie ofrece diversas formas y niveles de acceso a la lectura.

Desde el punto de vista de la crítica filosófica, existe toda una dimensión de la obra que puede ser leída de forma particular, y que es constituida por el análisis de los autores con los que trabaja Barbaras. En esta dimensión, la minuciosidad y envergadura del trabajo crítico es notable y puede constituir, en sí mismo, una obra de referencia sobre el tema de la vida en la tradición fenomenológica. Desde luego, esta crítica se guía por un objetivo sistemático, constituido por la presentación de la filosofía propiamente barbarasiana. Sin embargo, el fin no entra en conflicto con los medios, y es una muestra de la inteligencia de esta obra el dejar el espacio para un pensamiento conjuntamente crítico y sistemático.

Sin embargo, es esta última dimensión, la de la filosofía barbarasiana en sentido positivo, la que configura la verdadera apuesta, alcance y originalidad de la obra. En tanto aparición de un pensamiento nuevo, su presencia parece delinearse a partir de la continuidad y la diferencia de presupuestos, problemas y conceptos dentro del campo filosófico.

En este sentido, puede ser leída como el resultado, absolutamente original, de un pensamiento riguroso de la obra merleau-pontiana. Barbaras es, sin duda, el mejor discípulo de Merleau-Ponty. Para quien esté familiarizado con la obra de éste, es evidente que Barbaras entendió, no sólo en qué sentido Merleau-Ponty tenía razón, sino sobre todo en cuál no la tenía en absoluto. La continuación del programa merleau-pontiano, en los términos en que puede ser leido en Introduction à une phénoménologie de la vie, es al mismo tiempo una completa reformulación del mismo. Dicho de otro modo, si las preguntas que guian este libro son de corte merleaupontiano (¿cómo es posible una donación subjetiva que no sea coincidencia?, ¿cómo es posible un movimiento negativo que, sin excluir la posición, no desemboque en una identidad?, ¿cómo es posible una correlación que sea originaria respecto de sus polos?, ¿cómo hay que pensar al sujeto de la experiencia para que el sentir sea posible?), las respuestas son completamente barbarasianas (donación como exceso sobre lo positivo, el deseo como movimiento, la correlación definida a partir de la vida, el sujeto del deseo).
Del mismo modo, nos resulta difícil no pensar en una estrecha relación del pensamiento de Barbaras con el pensamiento hegeliano. Desde nuestro punto de vista, esta fenomenología de la vida debe ser comprendida como una fenomenología también en sentido hegeliano, esto es, como el despliegue de una experiencia de la vida misma. En este sentido, la represión de la vida a la que remite la ontología de la época moderna puede ser comprendida efectivamente como una represión de la vida, superando, una vez más, la alternativa del genitivo subjetivo u objetivo ${ }^{5}$.

Siguiendo así en el orden de lo no dicho, ¿cómo pasar por alto, en el contexto de esta fenomenología de la vida, que es al mismo tiempo fenomenología del deseo, la referencia -0 , mejor, la falta de referencia- a la teoría psicoanalítica? Fuera de una discusión a pie de página en relación con la noción de represión originaria - de gran densidad por cierto-, no hay otra referencia a la teoría freudo-lacaniana del deseo. Parece legítimo preguntarse, pues, en principio, por qué no tematizar al menos su exclusión, del mismo modo en que es tematizada, por ejemplo, la exclusión de la biología para determinar el sentido de ser de la vida. Sin embargo, esta pregunta se revela inmediatamente otra pregunta, cuya respuesta no corresponde, en sentido estricto, a la obra sino a sus lectores: ¿es posible, en efecto, establecer alguna suerte de diálogo entre algo así como una meta-física del deseo y la meta-psicología en sentido freudo-lacaniano?

De esta forma, Introduction à une phénoménologie de la vie nos abre, por una parte, a una reflexión sobre el modo de incorporar la crítica y la historia de la filosofía al campo de su realización concreta. Por otra, a un pensamiento nuevo que intenta pensar con todo rigor el fenómeno de la vida y del deseo reconfigurando, de esta forma, todo un campo de problemas. Pero nos abre también a la operación misma que pone en acto a través de este doble movimiento, y que no es otra que la mismísima actividad filosófica.

En efecto, si al comienzo de este trabajo remarcábamos, en el orden de las preocupaciones generales, una falta de reflexión sobre la noción de vida, la situación no se presenta de un modo muy diferente en el campo de la cultura. Así, frente al enorme espacio académico y mediático que hoy ocupan corrientes del pensamiento que suponen una noción de vida no siempre interrogada en su especificidad, la 
obra de Barbaras nos recuerda el lugar y la función propia de la actividad filosófica: el de una verdadera reflexión.

Una verdadera reflexión no es simple. Supone interrogar un concepto a partir del mundo en el que se presenta, pero también trascenderlo para poder comprender su sentido dentro de la totalidad. Supone desplegar un enorme trabajo crítico, conceptual y teórico para no dejarse atrapar en presuntas evidencias. Supone comprender la estructura metafísica en la que una noción se sustenta -estructura que obliga a explicitar el sentido de ser del mundo en el que esta noción aparece-, para poder llegar luego a su uso empírico. De lo contrario, ni uno ni otro podrán nunca comprenderse. Procediendo de este modo, la obra barbarasiana, reflexión sobre la vida del hombre, sujeto del deseo, se nos revela en toda su originalidad y pertinencia.

\section{NOTAS}

1 Barbaras, Renaud, Introduction à une phénoménologie de la vie. La obra que citamos a lo largo del artículo, y cuya fecha de publicación está prevista para febrero 2008 por la editorial J. Vrin, corresponde a la version manuscrita, a la que pudimos tener acceso gracias a la enorme gentileza del Dr. Barbaras.

2 La introducción del término "vivir" (vivre, en francés) surge de la búsqueda del espacio común de lo que, en alemán, aparece bajo los términos de Leben y Erleben. Tanto el francés como el español guardan en el "vivir" (vivre) la polisemia fundamental de los dos términos alemanes, que constituirán, sin embargo, una guía de lo que Barbaras denomina la univocidad y la equivocidad del vivir respectivamente.

3 Es importante señalar que la fenomenología barbarasiana de la vida se encuentre bien a resguardo de una perspectiva vitalista de la vida, en cualquiera de sus versiones. Al impugnar la posibilidad de la vida como principio positivo y teleológico, como inmanencia, y como conservación y acrecentamiento de sus condiciones de existencia, Barbaras deja de lado con toda elegancia una tradición que, de modo amplísimo, puede rastrearse de Bergson a Deleuze pasando por Nietzsche o, incluso, Simmel.

4 Esta perspectiva no debe confundirse con la de un "deseo metafísico", como podría ser el caso levinasiano. Como señala Barbaras, Levinas pierda la esencia del deseo al remitirlo a un orden otro que el del mundo terrestre y sus objetos deseables, dejando para este mundo sólo el campo de la necesidad.

5 De manera más específica, sería una tarea interesante a realizar, y aún en el marco de la relación entre los dos tipos de fenomenologias, un estudio de la noción de límite que atraviesa la obra barbarasiana, tomado en el marco de un movimiento de la negatividad que no remite a una identidad, a partir de las categorias hegelianas de la diferencia, para poder establecer así sus posibilidades de enriquecimiento mutuo.

Aceptado: 8 de enero de 2008 